\title{
Associations between maternal-child dietary vitamin D and calcium intakes in UK male and female adolescents aged 14-18 years
}

\author{
T. Smith, L. Tripkovic, K. Hart and S. Lanham-New \\ Department of Nutritional Sciences, School of Biosciences and Medicine, Faculty of Health and Medical Sciences, \\ University of Surrey, Guildford, Surrey, GU2 $7 X H$
}

Significant relationships between parent-child dietary intakes have been reported previously, with mother-child (M-C) correlations stronger than father-child correlations ${ }^{(1)}$. Additionally, mother-daughter (M-D) correlations may be stronger than for mother-son $(\mathrm{M}-\mathrm{S})$ pairs $^{(1)}$. There is little data relating to maternal influence on dietary vitamin $\mathrm{D}$ and calcium intakes in male or female adolescents, a crucial phase of rapid bone accrual and development of peak bone mass ${ }^{(2)}$.

The aim of this analysis was to examine associations between vitamin D and calcium intakes of mothers and adolescents (14-18 years) and specifically between M-D and M-S pairs recruited onto a 20 week vitamin D randomised controlled trial as part of the EU funded ODIN Project (Food-based solutions for Optimal vitamin D Nutrition and health through the life cycle). A total of $55 \mathrm{M}-\mathrm{C}$ pairs completed a Food Frequency Questionnaire at baseline to assess habitual vitamin D and calcium intakes from food sources only.

A significant, positive correlation was found between $M-C$ vitamin $D$ intakes $(r=0 \cdot 36, p=0 \cdot 007)$ and $M-C$ calcium intakes $(r=$ $0 \cdot 32, p=0 \cdot 016)$. When M-D $(n=32)$ and M-S $(n=23)$ pairs were investigated separately, a stronger and more significant positive correlation was identified for M-S versus M-D pairs for intakes of vitamin $D(r=0.41, p=0.05$ and $r=0.33, p=0.063$ respectively). However, for calcium intakes, correlations were not significant nor different between M-D and M-S pairs. Dietary vitamin D and calcium intakes for mothers and adolescents were divided into tertiles of intakes and chi-square test performed. Chi-squared test indicated significant associations between tertiles of vitamin D intakes in M-C and M-S pairings $\left(\chi^{2}=12 \cdot 52, p=0.014\right.$ and $\chi^{2}=9.89, p=0.042$ respectively) but not for M-D tertiles of intake. However, no significant associations were found for M-C, M-S or M-D tertiles of calcium intakes $\left(\chi^{2}=7 \cdot 52, p=0.111 ; \chi^{2}=4 \cdot 60, p=0 \cdot 331 ; \chi^{2}=6 \cdot 92, p=0 \cdot 140\right.$ respectively).

Therefore, contrary to previous studies citing stronger relationships between intakes of gender matched parent-child pairs ${ }^{(1)}$, the present analysis suggests that mothers may influence different types of food choices in their adolescent sons compared to their adolescent daughters, with a subsequent differential impact on vitamin D and calcium intakes. Stronger associations for dairy intakes between mothers and their adolescent daughters compared to adolescent sons have previously been reported ${ }^{(3)}$. This maternal influence may be important in the adolescent age group as $16 \%$ of UK females and $11 \%$ of UK males $11-18$ years have calcium intakes below the Reference Nutrient Intake (RNI) ${ }^{(4)}$. There is currently no UK RNI for vitamin D, although dietary intakes are low amongst both adolescents and adults at between 2 and $4 \mu \mathrm{g} / \mathrm{day}^{(4)}$. Sub-optimal calcium intakes during adolescence, alongside inadequate vitamin D intakes and status, has the potential to prevent maximum peak bone mass being achieved which could consequently lead to an increased risk of fracture and osteoporosis in later life ${ }^{(2)}$. Further analysis will investigate the potential behaviours underlying these relationships and how any positive associations may be manipulated to improve adolescent dietary patterns and nutrient status, particularly with regard to key but often inadequate nutrients such as vitamin D and calcium.

1. Wang et al. (2011) J Epidemiol Community Health, 65, 177-189.

2. Henry et al. (2004) Osteoporos Int, 15, 263-273.

3. Hanson et al. (2005) Public Health Nutr, 8, 77-85.

4. Bates et al. (2014) Public Health England. 